\title{
Integration of an Electron Cyclotron Imaging diagnostic system on the WEST tokamak
}

\author{
Roland Sabot ${ }^{1, *}$, Yoonbum Nam ${ }^{1}$, Cyril Brun ${ }^{1}$, Didier Elbèze ${ }^{1}$, Frédéric Faisse ${ }^{1}$, Laurent Gargiulo ${ }^{1}$, Minwoo Kim ${ }^{2}$, \\ Woochang Lee ${ }^{3}$, Philippe Lotte ${ }^{1}$, Hyeon K. Park ${ }^{2}$, Gunsu Yun ${ }^{4}$ and WEST team \\ ${ }^{1}$ CEA, IRFM, F-13108 Saint Paul-lez-Durance, France. \\ ${ }^{2}$ Ulsan National Institute of Science and Technology, Ulsan 44919, Korea \\ ${ }^{3}$ National Fusion Research Institute, Daejeon 34133, Korea \\ ${ }^{4}$ Department of Physics, Pohang University of Science and Technology, Pohang 37673, Korea
}

\begin{abstract}
An Electron Cyclotron Emission Imaging (ECEI) diagnostic system for the WEST tokamak has been developed under the UNIST-WEST collaboration. This diagnostic system is designed to overcome accessibility and thermomechanical constraints for long pulse operation. The first O-mode channel will be installed in the first trimester of 2019 to probe the low field side (LFS) of the WEST plasma. Two large metallic reflective mirrors are installed inside the duct which is being used for maintenance access. They are suspended on a rail to facilitate mirror manipulation. The ex-vessel optical system (lens, detection array, etc.) is housed in a compact optical enclosure that fits in a tight free space between the port flange and tokamak access lobby. The design emphasized reproducibility of the precise alignment between in-vessel mirrors and optical enclosure since the both elements must be removed during shutdown period for maintenance access. The overall optical system was fully tested at UNIST last year. The test results demonstrated that the imaging optics can full access at any radial position on the LFS. The 2D beam pattern measurements were consistent with the design values.
\end{abstract}

\section{Introduction}

WEST (Tungsten (W) Environment in Steady-state Tokamak) is transformation of the Tore Supra tokamak plasma in an X-point divertor configuration. WEST aims to test ITER divertor technology for high heat and particle fluence during long pulse operation [1]. Internal coils were installed to obtain flexible equilibrium: lower, upper or double X-point configuration. All carbon plasma facing components (PFC) were replaced with tungsten surface (W monoblock and carbon tiles coated with tungsten). The stainless steel water-cooled panels protecting the vacuum vessel were refurbished to cope with the new elongated configuration. The first WEST plasma was commissioned in 2016 and the divertor plasma configuration was obtained in 2017.

Control of magnetohydrodynamic (MHD) instabilities and metallic impurity transport is an essential step to sustain long discharges. Microwave imaging instrument offering a 2-D visualisation of the MHD as well as turbulence phenomena inside the plasma gives a unique insight of instability characteristics. Electron Cyclotron Emission imaging (ECEI) diagnostic system, first developed in the 90's, images the temperature fluctuations $[2,3]$. It has proven extremely powerful for understanding the physics of sawtooth crash [4,5], the structure of ELMs [6] or fast particles induced instabilities [7].

An ECEI system developed through the UNISTWEST collaboration will be installed during the first 2019 trimester. The ECEI will complement the set of diagnostics dedicated for fluctuation measurements [8]. $2 \mathrm{D}$ images of the temperature and density fluctuations induced by MHD instabilities could thus be obtained simultaneously [9].

This paper describes innovative approaches to integrate the ECEI optics on WEST. Although the WEST diagnostic relies on the ECEI principles, introduction of the large reflective optics to be positioned inside the manaccess port, which induce new constraints on the optics design compared to standard ECEI. The solution of both the in-vessel mirrors and compact ex-vessel optics was proposed to overcome the constraints in WEST will be described in part 2. The integrated optical system was fully tested last year at UNIST, Korea; the laboratory test results along with the alignment procedure will be presented in part 3 . The article concludes that although the ECEI implantation on large steady-state tokamaks is challenging, innovative solutions can overcome the integration issues to get the unrivalled information provided by $2 \mathrm{D}$ images of temperature fluctuations.

\section{WEST ECEI characteristics}

\subsection{Implantation in the man access port}

The ECE diagnostics is based on emission of the electrons around the magnetic field lines to measure the local electron temperature [10]. In the ECEI system, the single

* Corresponding author: roland.sabot@ecea.fr 
antenna of the conventional 1D ECE is replaced by an array of antennas equivalent to the layers of radiometers stacked vertically [3]. To guide the emission beam into the plasma to the order of $1-2 \mathrm{~cm}$ vertical and radial direction, the ECEI system needs a large access window and large optics to focus the emission beam.

Having a large vacuum window on WEST is very difficult due to the accumulative plasma radiation during long pulse discharge. Moreover, diagnostic ports were almost fully occupied. The only option was the maintenance access port where no diagnostic were implemented because of the tangential view to the plasma and a flange at several meters from the plasma edge. However, these drawbacks shadow the radiation fluxes and thus soften the thermomechanical constraints on the large window.

A preliminary design by $\mathrm{Y}$. Nam et al [11] demonstrated a feasibility of ECEI system using an Omode with the in-vessel mirrors at midway to refocus and redirect the emission beam (see fig. 1).

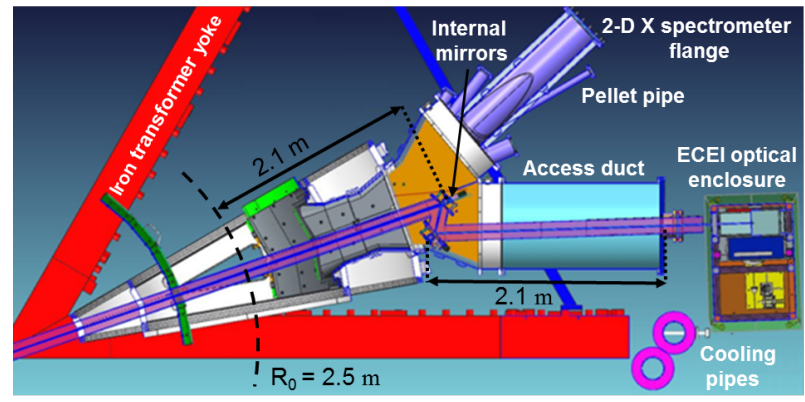

Fig. 1. ECEI beam path (purple colour). Two in-vessel mirrors are installed in the $\mathrm{Y}$ duct of the Q5A port shared by the maintenance access (bottom), the pellet injector and the 2D X spectrometer diagnostic (top). The ECEI beam is show in purple with the line of sight in blue.

Implementation of the current design in WEST presents several issues. Most ECEI diagnostics are located close to the window and benefit from the radial view. Here, the optics, usually composite lens, can be installed outside the vacuum vessel. In contrast, the in-vessel mirrors would be the first optical elements of the WEST ECEI system. The tight space for the ex-vessel optics made the integration of the focusing lens and detection array challenging. The available space between the flange and the access lobby was of the order of $1 \mathrm{~m}^{2}$ (fig 2).

Interference was another concern for the design because the ECEI diagnostic system would block the maintenance access for maintenance. The ex-vessel optics had to be removed to connect the access lobby (few meters long) to the torus during maintenance period. Also the in-vessel mirrors should be designed so that they can be displaced for the passage of maintenance crew. Moreover, both operations should have a marginal impact (few hours delay) on the first entrance inside the vessel.

Repositioning of the optics after each operation should also be guaranteed to avoid re-alignment effort after the maintenance period.

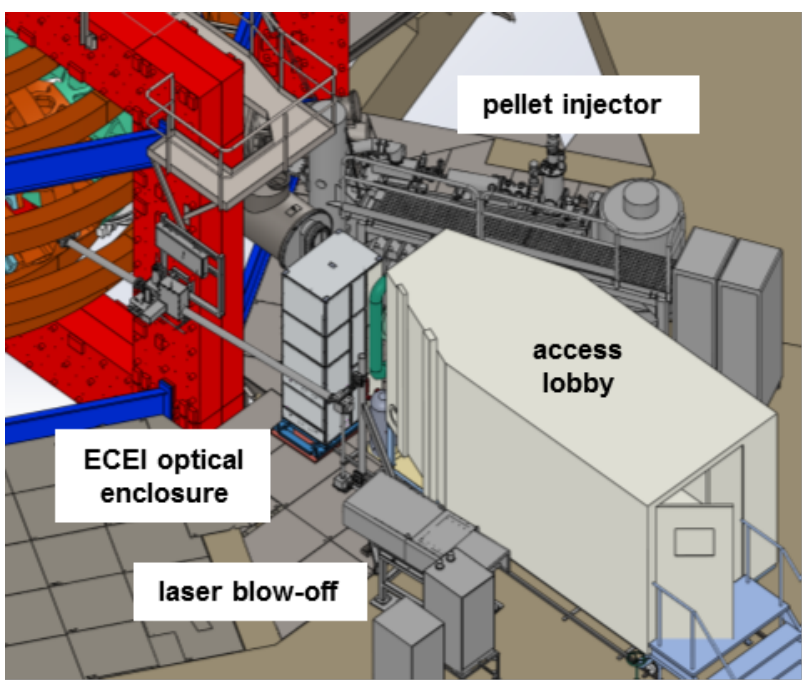

Fig. 2. View of WEST Q5A sector with the main elements showing the ECEI optical enclose, the access lobby and the pellet injector. The laser blow-off system is connected to the neighbouring sector. The X-ray imaging crystal spectrometer (not shown) is now installed on the right side of the pellet injector.

\subsection{In-vessel optics}

\subsubsection{In-vessel-mirrors}

The in-vessel mirrors are implemented in the $\mathrm{Y}$ duct at about $2.5 \mathrm{~m}$ from the plasma centre (fig 1). The design sought to maximize the mirror height $(\mathrm{H} \sim 59 \mathrm{~cm})$ to perverse good beam properties at all vertical position. The width was restricted to $20 \mathrm{~cm}$ (external width $27 \mathrm{~cm}$ ) to avoid self-shadowing. The first mirror from the plasma has a horizontal curvature $(\mathrm{R}=4.0 \mathrm{~m})$ and the second one is vertically curved $(R=3.5 \mathrm{~m})$ (see fig $3 \mathrm{a}$ ).

During the maintenance, the mirrors will remain inside the vacuum vessel to avoid the radiation and safety controls imposed on all elements exiting from the vacuum vessel. The mirrors on the rail track will be moved on the right to provide the path for crew to the vessel (see fig $3 \mathrm{~b}$ ). To facilitate mirror manipulation, the mirrors are suspended on the rails. The rails are similar to those installed in the WEST Articulated Inspection Arm (AIA) [12]. They can withstand machine baking and they have shown reliable operation under vacuum. Although the mirrors will not be manipulated under the vacuum, reliability of manipulation is a key safety issue.

To preserve the alignment after manipulation, the mirrors are mounted on a shaft with two pivot points. The top one frees the rotation and the displacement along the rail (see fig 4a). Reproducibility of the repositioning is guaranteed with a pin. Fine orientation of tuning $\left( \pm 2^{\circ}\right)$ and lateral position $( \pm 2 \mathrm{~cm})$ is done using the second pivot point at the centre of the mirror (see fig $4 \mathrm{~b}$ ). 

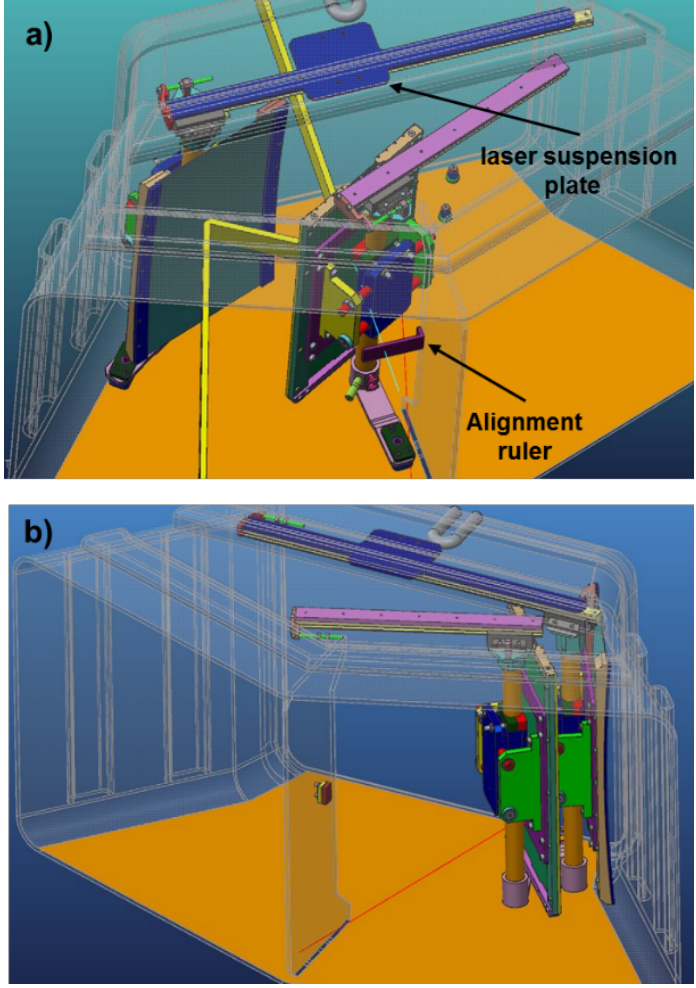

Fig. 3. Mirror positions during plasma operation (a) and during maintenance (b). Elements for alignment are also show (see 3.2)

The mirrors are attached to the middle of the shaft to ensure free and symmetric dilation during long pulses. The plasma radiation can heat the first mirror. Thermomechanical analysis predicts that the temperature at the centre of the first mirror would rise up to $430^{\circ} \mathrm{C}$ at the end of the reference WEST long pulse $(1000 \mathrm{~s}, 9 \mathrm{MW}$ of RF heating). The thermal expansion remains small and the optical quality of the microwave beam is not affected. Water cooling of the first mirror is thus not required.
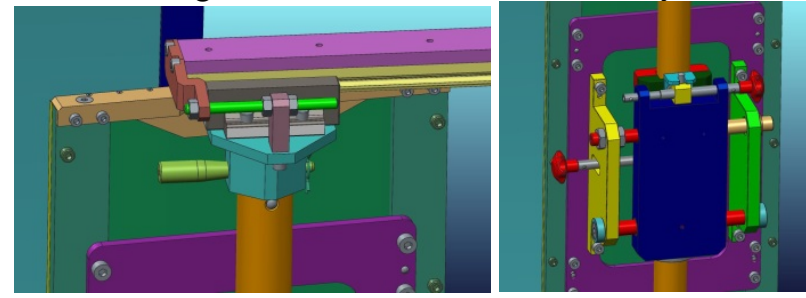

Fig. 4. Details view of the top pivot point (a) and fine tuning mechanisms (b).

\subsubsection{Angle of sight}

The magnetic ripple field in WEST reaches over $2 \%$ at the outer edge of the plasma. To minimize the broadening of the ECE channel due to the toroidal extension of the beam, a sight angle as close as possible to the middle of the port (lowest ripple variation) was desirable. The ECEI sight angle could not be lower than $1^{\circ}$ from the middle to avoid shadowing diagnostics installed in the port. As shown in the figure 3 , the ripple-induced channel overlapping at $1^{\circ}$ is negligible even at the edge. A second sight angle at $1.2^{\circ}$ can be used to widen further the pellet camera view.

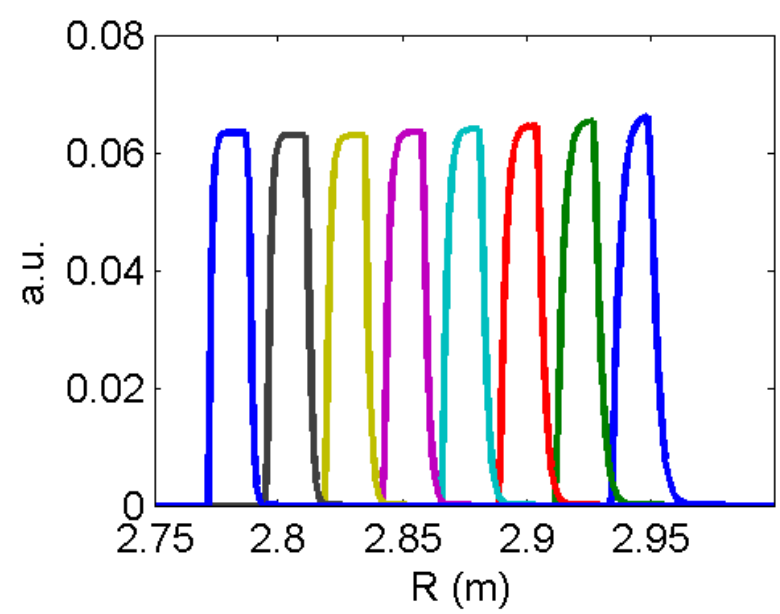

Fig. 5. Channel overlapping at the edge due to the ripple for the reference $1^{\circ}$ sight angle. The toroidal waist of the ECEI beam is $6 \mathrm{~cm}$ and the channel separation is $\Delta \mathrm{F}=900 \mathrm{MHz}$.

\subsection{External optics}

\subsubsection{Optical enclosure}

The housing for external optics and detection system had to fit in a limited space between the port and access lobby. Benefiting from the height of the WEST equatorial plane ( $2.5 \mathrm{~m}$ above the ground floor), the imaging optical components are vertically aligned inside the compact $(0.8$ $\mathrm{m} \times 1.2 \mathrm{~m} \times 2.5 \mathrm{~m}$ ) enclosure.

The lens at the optical enclosure entrance will first refocus the beam to the detectors (see fig 6). The $45^{\circ}$ mirror will reflect the beam downward. Dichroic plates will reject image frequencies. The polarization is then rotated to match with the antenna pattern of the detector array. The focal position of the beam in the plasma will be set by the position-controlled lens. The last lens focuses the elongated beam to the detector array made of two rows of 12 mini-lenses and mixers [13]. Extra effort was invested in design to make the imaging and local oscillator as compact as possible. In particular, a couple of aspheric lenses was used to shorten the optical path length of the LO optics to $\sim 0.5 \mathrm{~m}$ [14]. The design includes provision for a beam splitter for high field side (HFS) view. Notch filter will be inserted if the ECRH gyrotrons at $118 \mathrm{GHz}$ resume operation.
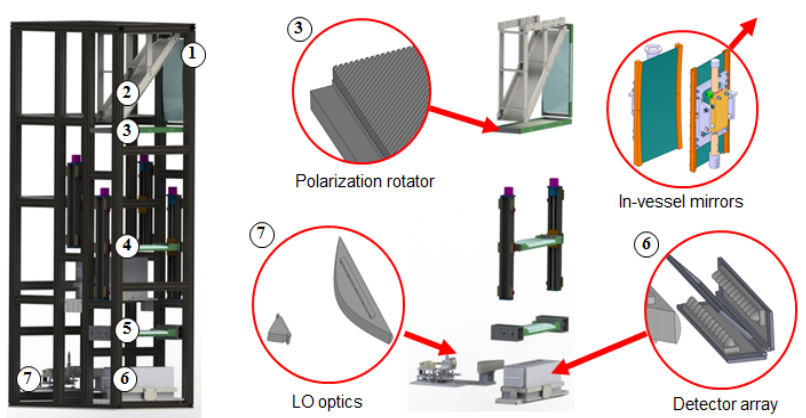

Fig. 6. Optical enclosure: (1) entrance lens, (2) $45^{\circ}$ mirror, (3) polarization rotator, (4) focusing lens (moveable), (5) collimation lens, (6) detector array box (7) LO optics. 
All elements were delivered to CEA in April 2018. Due to its height, the optical enclosure will be lifted by the torus hall crane and it will be placed in a dedicated area inside the torus hall during maintenance period.

\subsubsection{Optical enclosure supporting frame}

To ensure precise alignment after each reinstallation, the optical tower will be mounted on a supporting frame that enables to adjust, the lateral position, the orientation, the height and tilt of the optical enclosure (see fig 7). The supporting frame will be removed with the optical enclosure during maintenance period.

The optical enclosure will rest on dedicated plates fixed to the metallic beams below the ground floor. Socket ball like feet will guarantee the required reproducible positioning of the supporting frame after each manipulation.

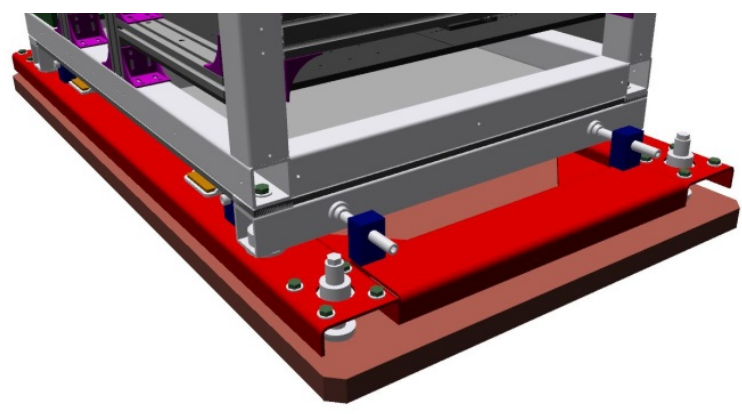

Fig. 7. Optical enclosure supporting frame. Screws and bolt allow tuning the position, orientation, height and tilt of the enclosure with respect to the ECEI beam.

\subsection{Control and acquisition cubicles}

Three cubicles in the basement below the ground floor house the IF electronics, the control and data acquisition system. The cubicles are assembled side by side. They are shielded against the electromagnetic and radiofrequency noises. The power supply for both channel will be installed in the central cubicle and each side cubicle will house the electronics and acquisition of one channel. 24 low loss $20 \mathrm{~m}$ long cables (True Blue 290 model from Teledyne) will connect the optical enclosure to the cubicles.

The diagnostic is operated by an commercial personal computer running LINUX OS connected to the WEST control and data acquisition network. Diverse devices in the cubicles or in the optical enclosure such as the LO frequency synthesizer, the step motors etc. are connected to the PC though a local network. The WEST timing network provides the time-stamping and event triggers for starting the acquisition.

The 192 channels are recorded by 6 acquisition units with 32 channels each at a sampling rate up to $2 \mathrm{Ms} / \mathrm{s}$ (ACQ132CPCI from D-tAcq), similar to KSTAR acquisition [15]. Up to $10 \mathrm{~s}$ at $500 \mathrm{kS} / \mathrm{s}$ can be recorded. The acquisition can be split in several triggers for synchronized measurements with other fast acquisition diagnostics during long discharges. To measure the background noise, the first trigger will start before plasma.

\subsection{Frequency range and plasma accessibility}

The first channel of the diagnostic will operate in the Wband covering the frequency range of $85-113 \mathrm{GHz}$. At the WEST nominal magnetic field $(B=3.7 \mathrm{~T})$, the diagnostic will fully cover the low field side (LFS) (see fig. 8). As the core MHD is a primary concern, the ECEI can probe the region beyond the plasma centre (from $\mathrm{R}=2.3$ at $\mathrm{B}=3.7 \mathrm{~T}$ ).

The diagnostic probes 8 radial positions with 900 $\mathrm{MHz}$ separation. The $7.2 \mathrm{GHz}$ IF bandwidth provides a radial coverage around $18 \mathrm{~cm}$ in the plasma centre and 24 $\mathrm{cm}$ at the edge. The diagnostic has 24 vertical channels to provide $8 \times 24$ pixel images of electron temperature fluctuations.

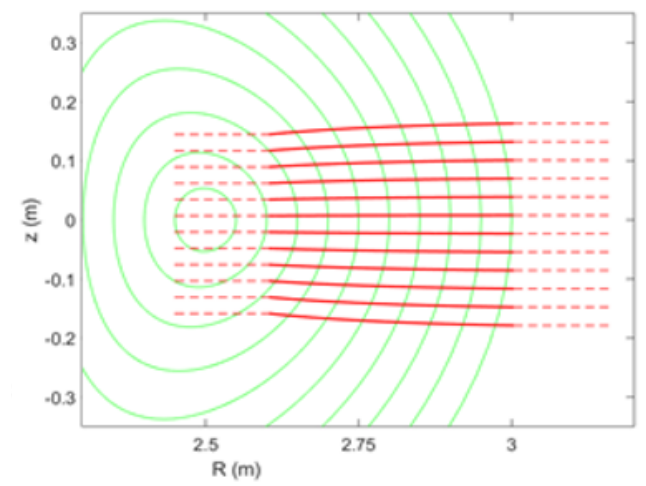

Fig. 8. First (LFS) channel accessibility at WEST nominal magnetic field $(3.7 \mathrm{~T})$.

A second channel at higher frequencies $(100-130$ $\mathrm{GHz})$ will be installed later to cover the inner side $(\mathrm{R}=2$ to $\mathrm{R}=2.6 \mathrm{~m}$ ) with some overlapping with the LFS channel in the plasma core.

\section{Optics properties and alignment}

\subsection{Imaging optics test}

\subsubsection{Experimental set-up}

The metallic mirrors were sent to UNIST, Korea in 2017 for integration test of the whole imaging optics. The beam path has been modified for laboratory test to facilitate measurements (fig. 9). These modifications have negligible effect on the beam property:

- the vertical $45^{\circ}$ mirror in the optical enclosure is not used and the optical elements are horizontally aligned

- the angle of the horizontally curved mirror have been adjusted to make the beam parallel.

- the beam source is placed at the detector box position, then the beam pattern at the plasma position is measured with a detector (reversed beam path).

The tests were performed at $90 \mathrm{GHz}$ using a frequency synthesizer and an active frequency multiplier (sextupler). Corrugated horn antenna and focusing lenses shaped beams with a $4.7^{\circ}$ divergence close to the $5^{\circ}$ divergence of the ECEI detection lens. More details on the test can be found in reference [16]. 


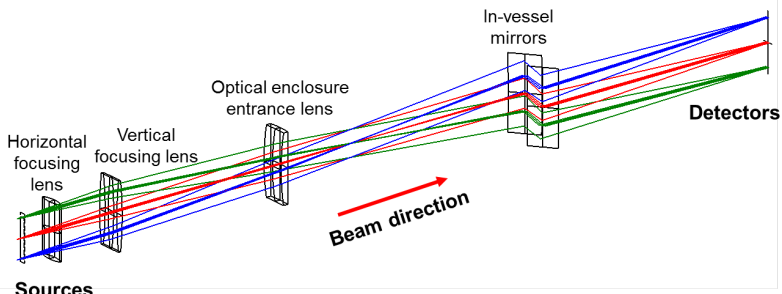

Fig. 9. Setup for integrated optics test for beam properties in laboratory

\subsubsection{Focusing capability test}

The focal position in the plasma is controlled by the vertical focusing lens: moving the lens toward the top optical enclosure lens moves the focus to the plasma edge. The beam pattern of the center channels $(\# 12,13)$ along the plasma position has been measured at the focus plane at the edge, at the mid-radius or at the plasma centre.

The imaging optics can focus on any radial position on LFS by controlling the vertical focusing lens. (2.5 $3.0 \mathrm{~m})$. The size at the focal position is around $1.6 \mathrm{~cm}$ and divergence is small enough for the beam size to be almost constant over the extension of the probing zone $(24 \mathrm{~cm}$ at the edge) as shown in figure 10 .

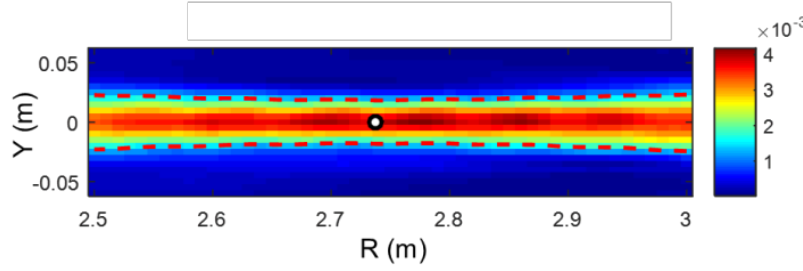

Fig. 10. Beam pattern (vertical direction) along the major radius. The white spot shows the position of the focal plane.

\subsubsection{Beam pattern of the different channels}

Beam pattern along the vertical direction was also measured for the same focal position: plasma edge, midradius and plasma centre. Results were compared to the design values (see fig. 11 for the mid-radius focus). The measured beam patterns are in good agreement with the design values. This demonstrates that the optical components are fabricated within the design precision.

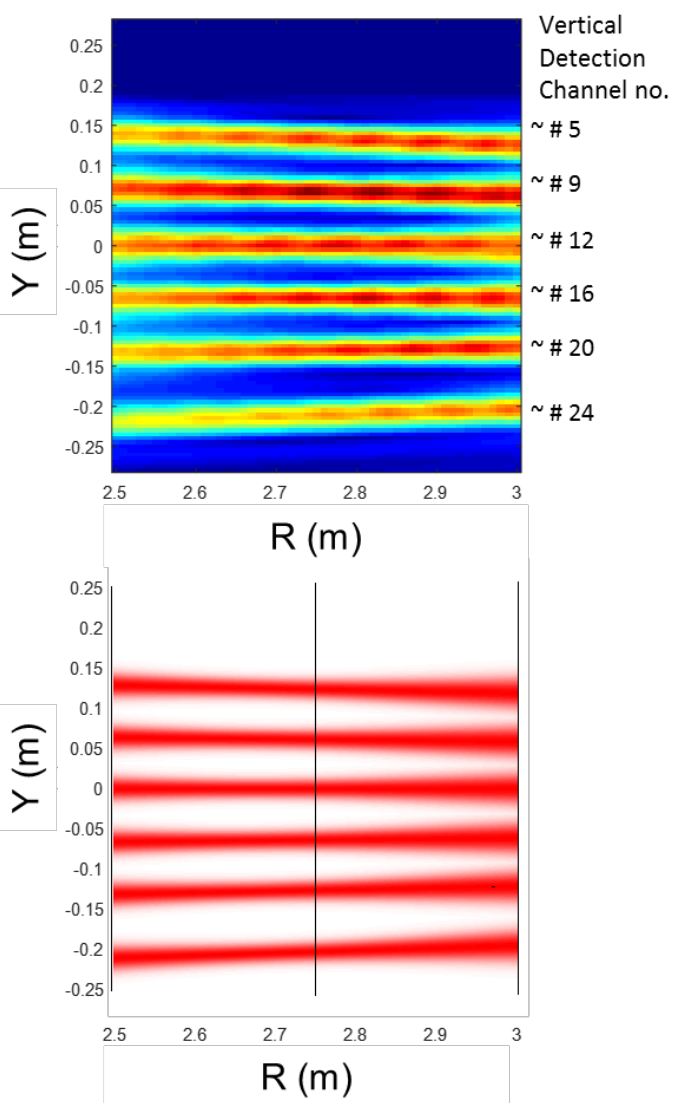

Fig. 11. Beam pattern (vertical direction) along the major radius. The white spot shows the position of the focal plane.

\subsection{Alignment procedure}

The WEST safety rule requires that all entry to the vacuum vessel must go through the access lobby. Hence, the alignment must be split in two phases: first, the invessel mirror alignment performed with the access lobby, then the optical enclosure after removing the access lobby.

The alignment will be performed with a cross laser (deviation less than $2 \mathrm{~mm}$ at $10 \mathrm{~m}$ ) to get both the equatorial and vertical planes. The laser will be suspended on the innermost rail. The first step is to align the laser with reference markers (horizontal and vertical planes) on the inner wall and back of the $Y$ duct (see fig 12). We will move the first mirror (M1) to get the laser beam at the mirror centre. The second mirror (M2) will then be set to its reference position and the angle of the first mirror will be adjusted so the laser beam points to the center of the M2 mirror. The M2 mirror will then oriented to get the laser beam on markers showing the position of the vacuum window middle inside the access lobby. The invessel alignment step is completed and the access lobby can be removed.

The height and position of the optical enclosure will be adjusted to get the laser beam at the centre of the $45^{\circ}$ mirrors. The lenses have to be removed because they are not transparent to the laser beam. By tuning the optical enclosure orientation and tilt angle, the detector array can be aligned with the $2 \mathrm{D}$ laser beam. Each lens starting from 
the bottom one will be installed and its position will be checked. The alignment ends after the installation of the last lens at the top of the optical enclosure. However, the alignment procedure will not yet be completed. The optical enclose must be removed and the access lobby must be connected. Then we can enter the vacuum vessel to remove the $2 \mathrm{D}$ cross laser.

During the first diagnostic alignment, we foresee to check that moving the mirrors back and forth or lifting up down the optical enclosure do not alter the diagnostic alignment.

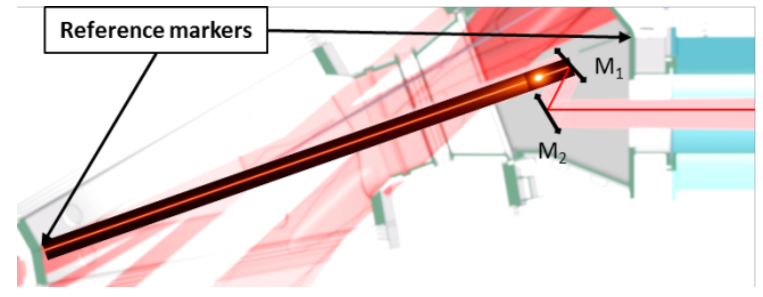

Fig. 12. Alignment set-up with a cross laser suspended on the innermost rail. Alignment order: laser, M1 position M2 position, M1 angle, M2 angle.

\section{Conclusion}

An optical systems for ECEI system that that can accommodate the hostile environment and constraint of long pulse discharge was designed and built for WEST. Innovate solutions have been developed to resolve the safety issue associated with the in-vessel access and tight space issues. Integration test demonstrated that the performances of the fabricated optics confirmed the precision of the design values. The ECEI system is now in a final assembly phase at Cadarache and will be installed on WEST during the 2018-19 winter shutdown.

The installation of the ECEI diagnostic on the next generation of devices would be much more challenging (thermomechanical stresses, vacuum window and radiation issues). However, the WEST ECEI demonstrates that some of these issues can be overcome in order to obtain the unrivalled value of 2-D images of temperature fluctuations for MHD and turbulence studies.

This diagnostic development was supported by a grant from the Région Sud Provence-Alpes-Côte d'Azur of France under Contract DEB 15-1376 and by the NRF of Korea under Contract Nos. NRF-2014M1A7A1A03029881 and NRF2014M1A7A1A03029865.

\section{References}

1. J. Bucalossi, A. Argouarch, V. Basiuk, O. Baulaigue, P. Bayetti, M. Bécoulet, B. Bertrand, S. Brémond, P. Cara, M. Chantant, Y. Corre, X. Courtois, L. Doceul, A. Ekedahl, F. Faisse, M. Firdaouss, J. Garcia, L. Gargiulo, C. Gil, C. Grisolia, J. Gunn, S. Hacquin, P. Hertout, G. Huysmans, F. Imbeaux, G. Jiolat, M. Joanny, L. Jourd'heuil, M. Jouve, A. Kukushkin, M. Lipa, S. Lisgo, T. Loarer, P. Maget, R. Magne, Y. Marandet, A. Martinez, D. Mazon, O. Meyer, M. Missirlian, P. Monier-Garbet, P. Moreau, E. Nardon, S. Panayotis, B.
Pégourié, R. A. Pitts, C. Portafaix, M. Richou, R. Sabot, A. Saille, F. Saint-Laurent, F. Samaille, A. Simonin, and E. Tsitrone, Fusion Engineering and Design 86, 684 (2011).

2. R. P. Hsia, W. R. Geck, S. Cheng, W. -M. Zhang, C. W. Domier, and N. C. Luhmann, Review of Scientific Instruments 66, 834 (1995).

3. H. Park, C. C. Chang, B. H. Deng, C. W. Domier, A. J. H. Donné, K. Kawahata, C. Liang, X. P. Liang, H. J. Lu, N. C. Luhmann, A. Mase, H. Matsuura, E. Mazzucato, A. Miura, K. Mizuno, T. Munsat, Y. Nagayama, M. J. van de Pol, J. Wang, Z. G. Xia, and W.-K. Zhang, Review of Scientific Instruments 74, 4239 (2003).

4. TEXTOR team, H. K. Park, N. C. Luhmann, A. J. H. Donné, I. G. J. Classen, C. W. Domier, E. Mazzucato, T. Munsat, M. J. van de Pol, and Z. Xia, Phys. Rev. Lett. 96, 195003 (2006).

5. TEXTOR Team, H. K. Park, A. J. H. Donné, N. C. Luhmann, I. G. J. Classen, C. W. Domier, E. Mazzucato, T. Munsat, M. J. van de Pol, and Z. Xia, Phys. Rev. Lett. 96, 195004 (2006).

6. G. S. Yun, W. Lee, M. J. Choi, J. Lee, H. K. Park, B. Tobias, C. W. Domier, N. C. Luhmann, A. J. H. Donné, J. H. Lee, and KSTAR Team, Physical Review Letters 107, (2011).

7. B. J. Tobias, I. G. J. Classen, C. W. Domier, W. W. Heidbrink, N. C. Luhmann, R. Nazikian, H. K. Park, D. A. Spong, and M. A. Van Zeeland, Physical Review Letters 106, (2011).

8. R. Sabot, P. Hennequin, and L. Colas, Fusion Science and Technology 56, 1253 (2009).

9. R. Sabot, D. Elbèze, W. Lee, Y. Nam, H. Park, J. Shen, G. Yun, M. Choi, J.-C. Giacalone, T. Nicolas, C. Bottereau, F. Clairet, P. Lotte, and D. Molina, Comptes Rendus Physique 17, 1018 (2016).

10. I. H. Hutchinson, Cambridge Core (2002).

11. Y. Nam, W. Lee, G. S. Yun, H. K. Park, D. Elbeze, J. L. Segui, R. Sabot, and J. P. Chenevoix, Review of Scientific Instruments 83, 10E318 (2012).

12. J.-C. Hatchressian, V. Bruno, L. Gargiulo, D. Keller, Y. Perrot, P. Bayetti, J.-J. Cordier, J.-P. Friconneau, J. D. Palmer, and F. Samaille, J. Phys.: Conf. Ser. 100, 062031 (2008).

13. B. Tobias, X. Kong, T. Liang, A. Spear, C. W. Domier, N. C. Luhmann, I. G. J. Classen, J. E. Boom, M. J. van de Pol, R. Jaspers, A. J. H. Donné, H. K. Park, and T. Munsat, Review of Scientific Instruments 80, 093502 (2009).

14. Y. B. Nam, D. J. Lee, J. Lee, C. Kim, G. S. Yun, W. Lee, and H. K. Park, Review of Scientific Instruments 87, 11E130 (2016).

15. J. B. Kim, W. Lee, G. S. Yun, H. K. Park, C. W. Domier, and N. C. Luhmann, Review of Scientific Instruments 81, 10D931 (2010).

16. Y. Nam, in 45th European Physical Society Conference on Plasma Physics (Prague, 2018). 